
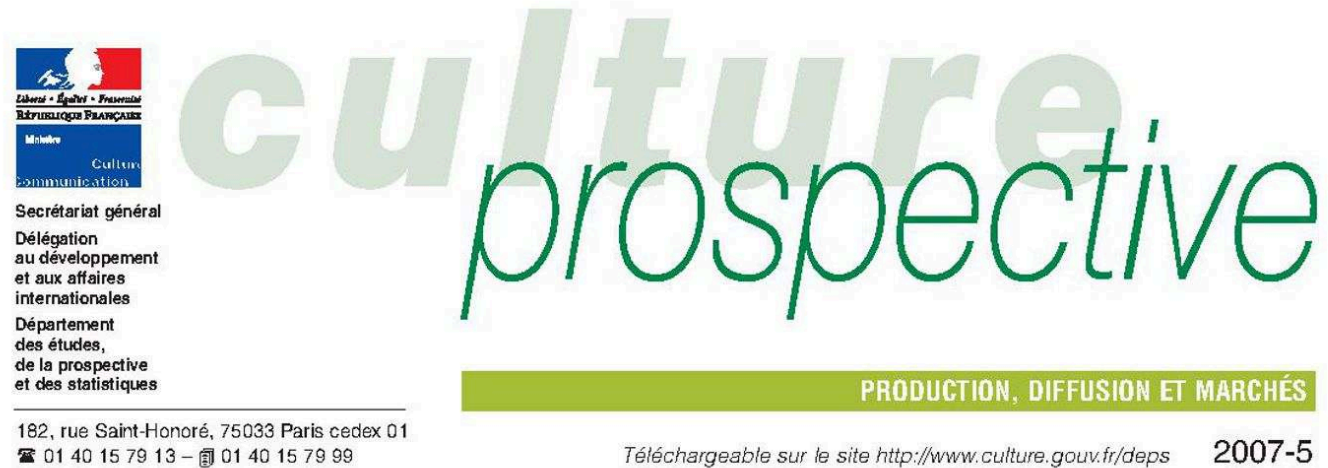

\title{
Organisation, processus et structures de la création
}

\section{Thomas Paris*}

\begin{abstract}
Avant-propos
«Industries créatives», «créativité », ces termes tendent à se substituer à «création». Récents dans la langue de l'économie de la culture, ils sont déjà au cour d'enjeux internationaux pour la définition du champ de la culture, de ses industries et, lors, des régulations possibles dans l'ordre commercial international ou communautaire. Ils traduisent surtout une réflexion partagée entre économie de la culture et économie industrielle et dénotent que l'organisation, les processus et les structures de la création sont au creur de la croissance de l'économie.

Fruit de l'appui conjoint du DEPS et de la DGE au ministère chargé de l'Industrie, ces premiers résultats de recherche éclairent les structures de la création - artistique comme industrielle -et leur fonction dans l'économie. Ils montrent le rôle clé de l'organisation, et que création et production culturelle sont une source de l'économie de l'immatériel et de la connaissance, ouvrant la voie à des actions publiques à la croisée de l'industrie et de la création.
\end{abstract}

Philippe Chantepie, DEPS

Grégoire Postel-Vinay, DGE à l'Industrie

\section{INTRODUCTION}

Organiser la création... paradoxe intrigant à double titre : parce que la création en tant qu' activité humaine est porteuse d'une connotation romantique et rattachée dans notre imaginaire à une notion d'auteur bien éloignée de celle d'organisation ; parce que les activités de création présupposent la mise en forme d'un construit mental original à réaliser. La division du travail, à l'origine du concept d'organisation, se heurte à cette difficulté intrinsèque de la communication : comment diviser le travail quand on ne sait l'exprimer qu'en le réalisant soi-même? Le paradoxe est d'autant plus fort que le monde académique a pris la mesure du poids des dimensions collective, organisée, conventionnelle des activités de création artistique ${ }^{1}$, et qu'il suffit d'observer empiriquement les industries dites «créatives» pour constater qu'elles reposent toutes sur des organisations qui, au-delà des individus créatifs, participent aussi de la créativité sur longue période.

Il s'agit de considérer la manière dont les activités de création s'inscrivent dans un univers organisé à tous les niveaux : ceux des entités les plus élémentaires organisées (univers collectifs), des entités juridiques (associations ou entreprises), des secteurs industriels, voire des nations. Car si les activités de création mettent en avant le talent et le génie individuel, elles reposent aussi sur des dispositifs et

\footnotetext{
* Chercheur en gestion au CNRs (Greg HeC et Crg École polytechnique, thomas paris@polytechnique.org). Ses recherches portent d'un côté sur le management de l'innovation, de l'autre sur l'economie de l'immatériel et le management des industries de la création. Auteur ou coordinateur d'ouvrages sur le droit d'auteur, la diversité culturelle dans le cinéma, l'economie de l'audiovisuel à l'ère de la convergence, il anime dinateur d'ouvrages sur le droit d'auteur, la diversité culturelle dans le cinéma, l'econome de l'audiovisuel à l'ère de la convergence, il anime
le séminaire Création à l'École de Paris du management. L'auteur exprime sa gratitude à ceux qui soutiennent ces travaux : (François Rouet et) le DEPS, (Michel Berry et) l'École de Paris du management, (Grégoire Postel-Vinay et) la Direction générale des entreprises au ministère chargé de l'Industrie, l'ensemble des intervenants et contributeurs au séminaire.

1. Howard S. BECKER, "Art as collective action", American Sociological Review, 1974, XXXIX(6), p. 767-776
} 


\section{Ministère Gulture

\section{Organisation, processus et structures de la création}

Artistic creation organisation, processes and structures

\section{Thomas Paris}

Éditeur : Département des études, de la prospective et des statistiques

Lieu d'édition : Paris

Année d'édition : 2007

Date de mise en ligne : 21 septembre 2015

Collection : Culture prospective

EAN électronique : 9782111398078

\section{OpenEdition}

\section{Books}

https://books.openedition.org

\section{Édition imprimée}

Date de publication : 1 novembre 2007

Nombre de pages : 16

\section{Référence électronique}

PARIS, Thomas. Organisation, processus et structures de la création. Nouvelle édition [en ligne]. Paris

Département des études, de la prospective et des statistiques, 2007 (généré le 15 février 2023).

Disponible sur Internet : <http://books.openedition.org/deps/876>. ISBN : 9782111398078.

(C) Département des études, de la prospective et des statistiques, 2007

Creative Commons - Attribution - Pas d'Utilisation Commerciale 4.0 International - CC BY-NC 4.0 


\section{RÉSUMÉS}

Les activités de création mettent en avant le talent individuel mais reposent aussi sur des dispositifs et des organisations. L'investigation soutenue par les ministères de la Culture et de l'Industrie a pris la forme d'un séminaire Création à l'École de Paris du management. Les résultats portent d'abord sur les processus de création et la manière dont les activités de création peuvent s'inscrire dans des projets et des entreprises dans un double mouvement de divergence et de convergence. On examine ensuite les liens entre création et structures en examinant comment est géré cet équilibre divergence-convergence et sont assumées des tâches spécifiques comme la gestion de la nécessaire abondance créative et, partant, l'échec de nombre de projets, la détection et la sélection des talents. La création s'en trouve à la fois encadrée en même temps qu'elle peut trouver de nouveaux espaces de développement.

While creative activities necessarily involve individual talent, they also need the support of systems and organisations. The investigation backed by the Ministries of Culture and Industry took the form of a seminar entitled 'Artistic Creation' held at the Paris School of Management. Its conclusions concerned, on the one hand, creative processes and the way these - in a dual movement of divergence and convergence - tie into projects and enterprises and, on the other, the connections between creation and structures. How can the divergence/convergence equation be resolved? How can specific problems such as management of the requisite creative abundance (and the corollary failure of numerous projects), talent discovery and talent selection be handled? Artistic creation is thus kept within limits while at the same time finding new room in which to develop.

\section{THOMAS PARIS}

Chercheur en gestion au CNRS (GREG HEC et CRG École polytechnique, thomas.paris@polytechnique.org). Ses recherches portent d'un côté sur le management de l'innovation, de l'autre sur l'économie de l'immatériel et le management des industries de la création. Auteur ou coordinateur d'ouvrages sur le droit d'auteur, la diversité culturelle dans le cinéma, l'économie de l'audiovisuel à l'ère de la convergence, il anime le séminaire Création à l'École de Paris du management. 

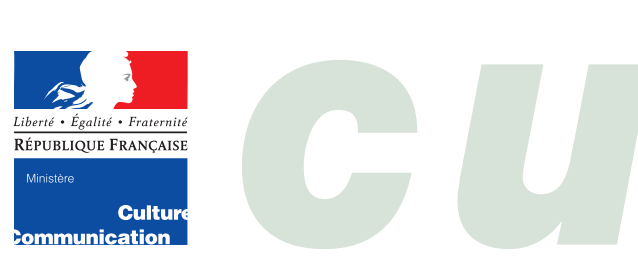

Secrétariat général

Délégation

au développement

et aux affaires

internationales

Département

des études,

de la prospective

et des statistiques

PRODUCTION, DIFFUSION ET MARCHÉS

\section{Organisation, processus et structures de la création}

\section{Thomas Paris*}

\section{Avant-propos}

"Industries créatives ", "créativité », ces termes tendent à se substituer à «création ». Récents dans la langue de l'économie de la culture, ils sont déjà au cour d'enjeux internationaux pour la définition du champ de la culture, de ses industries et, lors, des régulations possibles dans l'ordre commercial international ou communautaire. Ils traduisent surtout une réflexion partagée entre économie de la culture et économie industrielle et dénotent que l'organisation, les processus et les structures de la création sont au cour de la croissance de l'économie.

Fruit de l'appui conjoint du DEPS et de la DGE au ministère chargé de l'Industrie, ces premiers résultats de recherche éclairent les structures de la création - artistique comme industrielle - et leur fonction dans l'économie. Ils montrent le rôle clé de l'organisation, et que création et production culturelle sont une source de l'économie de l'immatériel et de la connaissance, ouvrant la voie à des actions publiques à la croisée de l'industrie et de la création.

Philippe Chantepie, DEPS Grégoire Postel-Vinay, DGE à l'Industrie

\section{INTRODUCTION}

Organiser la création... paradoxe intrigant à double titre : parce que la création en tant qu'activité humaine est porteuse d'une connotation romantique et rattachée dans notre imaginaire à une notion d'auteur bien éloignée de celle d'organisation; parce que les activités de création présupposent la mise en forme d'un construit mental original à réaliser. La division du travail, à l'origine du concept d'organisation, se heurte à cette difficulté intrinsèque de la communication : comment diviser le travail quand on ne sait l'exprimer qu'en le réalisant soi-même ? Le paradoxe est d'autant plus fort que le monde académique a pris la mesure du poids des dimensions collective, organisée, conventionnelle des activités de création artistique ${ }^{1}$, et qu'il suffit d'observer empiriquement les industries dites «créatives » pour constater qu'elles reposent toutes sur des organisations qui, au-delà des individus créatifs, participent aussi de la créativité sur longue période.

Il s'agit de considérer la manière dont les activités de création s'inscrivent dans un univers organisé à tous les niveaux : ceux des entités les plus élémentaires organisées (univers collectifs), des entités juridiques (associations ou entreprises), des secteurs industriels, voire des nations. Car si les activités de création mettent en avant le talent et le génie individuel, elles reposent aussi sur des dispositifs et

\footnotetext{
* Chercheur en gestion au CNRS (GREG HEC et CRG École polytechnique, thomas.paris@ polytechnique.org). Ses recherches portent d'un côté sur le management de l'innovation, de l'autre sur l'économie de l'immatériel et le management des industries de la création. Auteur ou coordinateur d'ouvrages sur le droit d'auteur, la diversité culturelle dans le cinéma, l'économie de l'audiovisuel à l'ère de la convergence, il anime le séminaire Création à l'École de Paris du management. L'auteur exprime sa gratitude à ceux qui soutiennent ces travaux : (François Rouet et) le DePs, (Michel Berry et) l'École de Paris du management, (Grégoire Postel-Vinay et) la Direction générale des entreprises au ministère chargé de l'Industrie, l'ensemble des intervenants et contributeurs au séminaire.

1. Howard S. BECKER, “Art as collective action”, American Sociological Review, 1974, XXXIX(6), p. 767-776.
} 
des organisations et peuvent être partiellement formalisables : les connaissances nécessaires à ceux qu'on appelle «talents » peuvent être partagées. Il existe des techniques et modes d'enseignement qui en permettent l'acquisition. Les organisations peuvent elles-mêmes être porteuses de créativité par le biais de règles, routines ou procédures, et elles peuvent prendre place dans un environnement plus ou moins fertile, porté par des structures sociales, juridiques, financières, culturelles, mais aussi par des dispositifs originaux capables de favoriser la rencontre, l'émergence, l'apprentissage...

L'analyse des dispositifs organisationnels, les «structures de la créativité », permet d'aborder les enjeux industriels de ce que des pays anglo-saxons désignent comme «industries créatives » et qui recouvrent tant les industries culturelles que la mode, la publicité, la grande cuisine, le design industriel... Enjeux économiques pour lesquels ces industries ou une partie d'entre elles sont tenues pour stratégiques ${ }^{2}$, mais aussi enjeux internationaux car, des définitions du champ de la créativité, dépendent les conditions des régulations spécifiques propres au champ culturel.

Plusieurs enjeux peuvent notamment être identifiés :

- la pérennisation des entreprises de création après leur fondateur, qui dépend de la transmission et de la capacité à formaliser l'organisation de la création ;

- la réduction des barrières à l'entrée pour stimuler le renouvellement créatif, qu'il s'agisse des créatifs ou des managers, le star system et l'inflation du prix des talents étant consubstantiels des industries de la création ${ }^{3}$;

- le renouvellement de l'action publique pour tenir compte du fait que la création n'est pas le fait de seuls talents, mais le fait de structures et de l'organisation de la création.

L'analyse de ces problématiques nécessite l'adoption d'une perspective de management stratégique, c'est-à-dire centrée sur la question organisationnelle et intégrant la dimension de la performance. Elle suppose d'autre part que l'activité de création est un élément structurant des réponses apportées en termes d'organisation, et que la création se faisant dans un continuum production-dif-

\section{Glossaire}

Création-Processus, acte par lequel un créateur conçoit une œuvre, un message publicitaire, un plat culinaire, un dessin de produit...

Industries créatives - Industries (au sens de «secteur » ou " filières ") dans lesquelles le produit final est un objet de création : audiovisuel, musique, édition, mode, design, publicité, gastronomie, spectacle, arts plastiques...

Créativité - «Performance » créative d'un groupe, d'une entreprise, d'une industrie, d'un territoire. C'est la qualité (quantitative et qualitative) de sa production de produits de création. - Appliquée à l'individu, capacité à avoir une production créative.

Talents - Dans un secteur créatif donné, individus présentant une aptitude notable à la création. Plus généralement, dans des secteurs connexes, individus présentant une aptitude particulière dans leur métier qui les rend peu substituables ou leur confère une certaine rareté (hauts potentiels dans l'industrie, sportifs de très haut niveau...). Dans les industries créatives, il s'agit en général aussi bien des créatifs que des individus qui font montre d'une capacité particulière à les détecter ou à les gérer.

Créatifs-Participants d'un acte de création, dès lors qu'il implique une division du travail.

Créateurs - Ceux qui ont la responsabilité de la création et qui sont reconnus comme les auteurs.

Structures de la création - Ensemble des dispositifs et institutions constitutifs dans un secteur donné d'un environnement qui favorise la création ou sa diffusion.

fusion, son organisation s'aborde depuis un niveau micro (entités élémentaires, projets ${ }^{4}$ ).

La synthèse des résultats porte en premier lieu sur les processus de la création et la manière dont les activités de création peuvent s'inscrire dans des projets et des entreprises, en second lieu sur le lien entre structures et création, soit la manière dont les structures existantes peuvent avoir un impact sur les produits de la création.

\section{LES PROCESSUS DE CRÉATION}

Les activités de création constituent, quel que soit le secteur, une catégorie homogène du fait d'un certain nombre de spécificités partagées qui les distinguent d'autres activités. Il s'agit d'activités dont le résultat n'est pas évaluable de manière objective et n'est pas exprimable autrement que par sa réalisation même, des activités dont les caractéristiques rentrent a priori en conflit avec les contraintes industrielles et économiques des organisations (voir tableau 1). Le management de la création consiste donc à faire cohabiter les processus de création avec les exigences et contraintes liées à l'organisation.

2. Maurice LÉVY et Jean-Pierre JouYET, L'économie de l'immatériel. La croissance de demain. Rapport de la Commission sur l'économie de l'immatériel, Paris, Ministère de l'économie, des finances et de l'industrie, 2006.

3. Françoise Benhamou, L'économie du star system, Paris, Odile Jacob, 2002.

4. Howard S. BECKER, Art Worlds, The University of California Press, 1982. 


\section{Les processus de création}

Aléas de l'inspiration (de l'incertain, de l'incontrôlable)

Abstraction des idées

Les talents, qui sont des individus particuliers, sont caractérisés par une certaine fragilité, du fait des aléas dans l'inspiration et dans la réception de leurs productions

Grande liberté de l'inspiration
Les mondes de l'organisation

Contraintes économiques : du fait des coûts fixes qu'elles ont à assumer, exigence de régularité dans la production

Division du travail et formalisation

Les talents, par leur rareté, sont extrêmement précieux pour les organisations qui exploitent leur travail

Contraintes - la ligne éditoriale - liées à leur image de marque et à leur clientèle, et fonctionnement

autour de routines tendant à formater leurs productions
Les activités de création ont d'autre part une dimension collective qui se traduit par le rôle, par exemple dans la création d'un livre ou d'un disque, de l'éditeur ou du producteur et, naturellement, dans le design ou le jeu vidéo. Mais malgré cette dimension collective, le résultat final reste la création d'un individu, au sens où elle lui est attribuée et où il en assume le résultat. Aussi, la capacité à articuler les dimensions collective et individuelle des processus de création apparaît-elle essentielle.

Selon cette approche, certains traits marquants ont été mis en évidence chez les créatifs et créateurs.

\section{Les deux temps du management de la création : divergence et convergence}

L'analyse de cas issus de différents secteurs met en évidence des régularités dans les processus de création qui reposent sur l'articulation entre des temps de divergence et des temps de convergence alternativement phases d'ouverture et de cadrage. Ces temps visent à «réaliser » petit à petit une idée à travers quatre étapes : inspiration ou production d'idées, cadrage ou directions pour guider le processus, mise en forme et validation (voir graphique 1). Ces étapes n'interviennent pas d'une

\section{Graphique 1 - Processus de création}

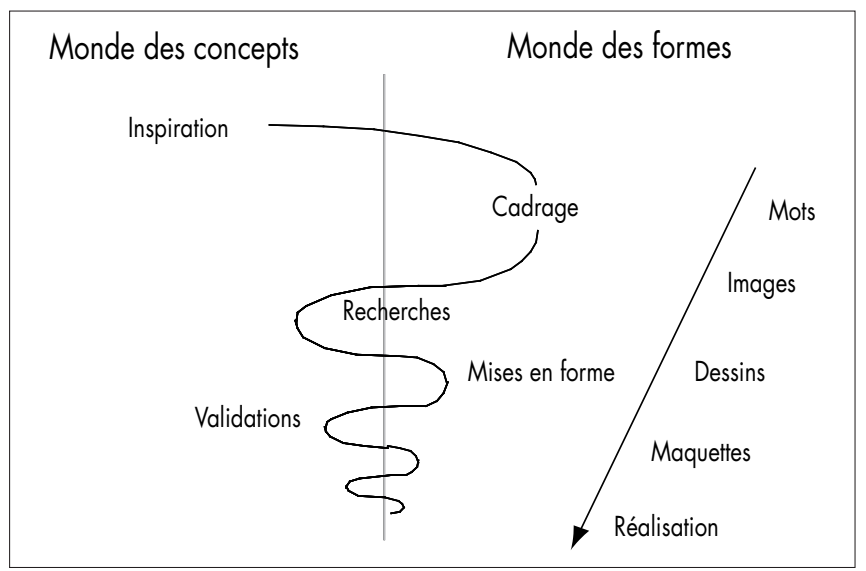

manière linéaire, chacune pouvant rétroagir sur celle qui la précède et faire évoluer l'idée. Réaliser une idée ne signifie pas parvenir à la transposer dans le monde des objets, mais parvenir à l'exprimer, car l'idée n'a pas d'existence autre que celle de sa mise en forme, contrairement à ce que peut laisser entendre une phrase comme « le réalisateur a le film dans sa tête ».

\section{Division du travail}

Les processus de création peuvent faire intervenir un ou plusieurs créatifs. Dans l'édition, en règle générale, l'auteur prend en charge la totalité des phases : de ce fait, inspiration, cadrage, mise en forme et validation ne sont plus clairement distincts mais se font en permanence. Néanmoins, d'autres modèles sont possibles: Alexandre Dumas était connu pour s'appuyer sur des «nègres », et s'il intervenait au niveau de l'inspiration, du cadrage et de la validation, il laissait la réalisation à d'autres. Dans la création de mode, le créateur - celui qui est identifié comme tel - intervient en début de processus et au niveau de la validation, tout le travail de mise en forme étant réalisé par d'autres. Ainsi, a minima, le cadrage initial et la validation finale sont-elles toujours prises en charge par celui qui assume la responsabilité de l'œuvre. Ce sont ces phases qui définissent la qualité d'auteur. Dans des cas extrêmes où la création est externalisée - par exemple dans le cas de licences dans la mode -, le signataire conserve le droit de regard final, la notion de créateur s'amenuisant au profit de celle de marque.

\section{Inspiration}

L'idée même d'inspiration - « on est ou on n'est pas inspiré »- sous-entend le caractère incontrôlable de cette phase, alors que les contraintes économiques imposent au contraire que cette phase soit «activée ». Dans ce but, certains dispositifs sont mobilisés pour favoriser l'inspiration: voyages, 
plongées dans des univers artistiques ou culturels, rencontres avec d'autres cultures ou champs de création... John Galliano, le créateur de mode de Christian Dior Couture, voyage avant chaque lancement de collection. Le chef Alain Senderens, outre les voyages, se renouvelle à travers des cours d'œnologie, des rencontres avec un maître-thé... Cette recherche d'ouverture et d'inspiration se retrouve au cœur du processus de création, dans la phase où ceux qui participent à la création doivent faire des propositions à partir du cadre imposé par le créateur. Les managers s'efforcent de maintenir la créativité des créateurs en faisant en sorte qu'ils s'ouvrent à d'autres cultures: les designers de Renault sont envoyés sur des événements culturels internationaux pour se confronter aux nouvelles tendances, les créateurs de jeux vidéo de Kalisto dans des expositions ou dans des musées...

Sans parler d'inspiration, le besoin de création peut s'inscrire simplement dans un flux régulier qui rythme le renouvellement: c'est le cas du système des collections et des défilés dans la mode. Il peut également être créé par les contraintes industrielles (les magasins à alimenter, la cherté des stocks qu'il faut gérer au plus près, les usines à servir) ou imposé par un changement - d'ordre technologique ou économique - dans les conditions des processus créatifs : les variations du cours des matières premières peuvent par exemple orienter les créations d'un restaurant, comme Alain Senderens décidant de travailler un produit en forte baisse sur le marché. Enfin, le besoin de nouveauté peut tout simplement être lié à un besoin normal de renouvelle- ment, de remise en cause, de dépassement... chez le créateur.

\section{Cadrage}

Du fait de la nature même de l'acte de création, la question du cadrage - c'est-à-dire la définition du périmètre dans lequel le créatif fera ses recherches - est délicate. Comment en effet exprimer la création que l'on veut réaliser sans la réaliser soimême ? Le créateur s'appuie sur l'arsenal de modes d'expression «simples » à sa disposition : mots, dessins, images... Chez Christian Dior Couture, ce sont des mots-clés énoncés par le créateur - « gothique chic » par exemple -, parfois illustrés par des images et des dessins, parfois accompagnés d'histoires. Les références peuvent aussi servir à préciser un cadre. Nicolas Gaume ${ }^{5}$ se souvient de celui qui fut défini pour le jeu Sombre Terre : «Créer un univers, inspiré de Jules Verne - cuivres, matériaux de récupération, vapeurs...-, de Méliès et de notre culture européenne: nous voulions reproduire l'image d'immenses cathédrales construites au milieu de maisons de torchis. »

Le cadre peut ainsi être orienté de plusieurs façons : sur les produits utilisés (matières, thèmes, couleurs...), les techniques, le résultat final (rythme, atmosphère...), sur la création même ou sur une cible de clients. Ainsi, dans le secteur de la parfumerie, un brief créatif ${ }^{6}$ est en général à l'origine de la création. Le cadrage, nécessaire pour favoriser la convergence vers la réalisation d'un produit, est également très précieux pour encourager la créativité des équipes. Pour Brigitte Romagné7, le

Tableau 2 - Formes et exemples de cadrage

\begin{tabular}{|c|c|c|c|c|}
\hline & \multicolumn{4}{|c|}{ Objets du cadrage } \\
\hline & $\begin{array}{l}\text { Matières premières } \\
\text { (produit, couleur...) }\end{array}$ & Techniques & $\begin{array}{c}\text { Résultat } \\
\text { (rythme, atmosphère...) }\end{array}$ & $\begin{array}{c}\text { Cible } \\
\text { de clients }\end{array}$ \\
\hline \multicolumn{5}{|l|}{$\begin{array}{l}\text { Outils de } \\
\text { formalisation }\end{array}$} \\
\hline Mots & $\begin{array}{l}\text { «Ris de veau » (grande cuisine) } \\
\text { «Cuivres, matériaux de récupération, } \\
\text { vapeur » (jeu vidéo) }\end{array}$ & $\begin{array}{l}\text { «Foie chaud» } \\
\text { (grande cuisine) }\end{array}$ & $\begin{array}{l}\text { « Gothique chic » } \\
\text { (mode) }\end{array}$ & $\begin{array}{l}\text { Brief créatif } \\
\text { (parfum) }\end{array}$ \\
\hline Illustrations & & & Boards (posters) (mode) & \\
\hline Références & & & $\begin{array}{l}\text { eur de barbe à papa " (pa } \\
\text { rs issu de Jules Verne » (je }\end{array}$ & \\
\hline
\end{tabular}

5. Nicolas Gaume est le créateur de Kalisto, studio de développement de jeux vidéo.

6. Le brief créatif définit l'objectif, le support, le concept, le ton de la création ainsi que le message à faire passer. Il correspond à la phase réflexion avant de passer à l'action (brief annonceur).

7. Brigitte Romagné a été successivement directrice du marketing et de la prospective pour une marque de parfums et de cosmétiques, puis a dirigé une équipe de nez en qualité de directrice de création. Elle forme les professionnels du marketing à la découverte des essences naturelles. Elle a publié plusieurs ouvrages sur les plantes et épices à parfums. 
manque de précision des briefs créatifs proposés par les marques de parfums aux créateurs se traduirait par un manque de créativité dans le travail des nez. Mais, Thierry Mugler, pour créer le parfum Angel, est parti du souvenir très personnel de l'odeur de la barbe à papa dans les fêtes foraines.

Dans chaque secteur, on trouve ainsi des créations de créateur et des créations plus ciblées sur des considérations marketing. Enfin, selon la nature des créations, et les sens qu'elles mobilisent, le cadre sera plus ou moins facile à formaliser. Dans la gastronomie ou la parfumerie, exprimer le résultat attendu à partir de mots et d'images sera plus difficile que dans le design.

\section{Mises en forme}

Le travail des équipes de création se fait à partir du cadrage proposé. La complexité des modes de réalisation et l'importance des coûts de développement rendent plus ou moins maniable l'outil dont dispose le créateur pour s'exprimer : quand il s'agit du pinceau ou du stylo, il conserve une relative mâ̂trise de l'adéquation entre sa vision et sa réalisation, alors que les modes de création exigeant la mobilisation d'outils techniques sophistiqués et d'équipes composites importantes accroissent cette « distance », comme le montre l'exemple de la réalisation d'un film et d'un jeu vidéo. Dans le film, grâce à l'écran de contrôle sur lequel il peut visualiser la position et le jeu des acteurs ainsi que le cadrage, le créateur peut les faire évoluer avant la prise de vue, alors que dans un dessin animé, il ne voit le résultat qu'une fois le travail réalisé et n'a donc plus la possibilité de modeler sa création.

En pratique, plus la distance est grande, plus il y a d'étapes intermédiaires dans la mise en forme, ces étapes s'appuyant sur différents objets qui se rapprocheront petit à petit du résultat final. Chacune donnant lieu à validation et/ou recadrage, elles permettent ainsi de préciser la vision du créateur. Plusieurs modalités de représentation interviennent dans la phase de création, certaines axées sur la forme - croquis, dessins, maquettes...-, d'autres ayant vocation à montrer la structure, notamment dans le cas des œuvres dramatiques - synopsis, scénario...

\section{Validation}

À chacune des étapes, le créateur, responsable de la création, valide ou non les différentes mises en forme qui lui sont proposées. Cette phase est parfois aussi une phase de sélection parmi les diffé- rents projets proposés. C'est le cas chez Renault où l'organisation du design automobile repose sur des concours organisés à chaque étape. La validation finale - final cut dans le langage du cinéma - reste le plus souvent la prérogative du créateur, fût-elle parfois partagée avec un manager, responsable du projet, investisseur financier ou garant d'une image de marque.

La modélisation générale des processus de création proposée ici correspond à l'ensemble des situations rencontrées dans des secteurs très variés. Mais des différences peuvent apparaître selon les secteurs, selon les entreprises, car les contraintes diffèrent en fonction du secteur, du créateur.

\section{Des spécificités sectorielles}

Trois facteurs expliquent les différences de déroulement du processus : l'incommunicabilité, la complexité de l'outil de production et la nature des contraintes de diffusion.

L'incommunicabilité est liée à la nature de la création et traduit le degré de difficulté à exprimer par les outils de communication usuels le résultat que le créateur souhaite obtenir. Cette difficulté sera ainsi beaucoup plus grande lorsqu'il s'agit de parfum, faisant appel à un sens pour lequel peu de mots existent, que dans le cas d'un ouvrage littéraire, dont le contenu peut s'exprimer à partir de mots.

La complexité de l'outil de formalisation de la création est relative aux techniques de création qui servent au créateur pour s'exprimer: la main du sculpteur et sa sculpture ou, dans le cas du cinéma, l'agencement complexe d'outils techniques, d'équipes composites aux compétences multiples et d'êtres humains. Dans le premier cas, les différentes étapes de la création seront étroitement liées, chaque mouvement de la main du sculpteur pouvant incorporer l'ensemble de ces phases alors que, dans le second, le travail est soumis à des contraintes économiques et organisationnelles.

En général, plus l'outil de production est complexe, plus le coût de développement à chaque étape sera important, et plus le passage à chacune sera contrôlé. Alors que le processus de divergenceconvergence est circulaire lorsqu'il s'agit d'une création réalisée directement par son créateur, il sera beaucoup plus scandé dans le cas où l'outil de production est un agencement complexe. Plus l'outil nécessite la mobilisation de compétences variées et d'outils techniques sophistiqués, plus le découpage du processus sera marqué, et plus il comportera d'étapes. 
Les contraintes de diffusion des créations relèvent de considérations industrielles. Lorsque le flux de créations nouvelles est régulier, une pression s'exerce sur les processus créatifs et leurs calendriers. En outre, lorsque l'entreprise intègre la création - notamment lorsqu'elle veut contrôler son image de marque -, l'existence d'une clientèle régulière bien identifiée fait que les contraintes de flux se répercutent beaucoup plus directement sur les équipes concernées. Dans ce cas, le cadrage initial peut être contraint par cette notion d'image de marque, alors que, dans d'autres situations, il est beaucoup plus ouvert.

\section{Des formes variées d'organisation industrielle de la création}

Les processus de création peuvent donc donner lieu à des formes d'organisation industrielle (ou chaînes de valeur) très variées : la création peut être intégrée ou non, la mise en mouvement du processus peut être impulsée par les créateurs eux-mêmes ou par des acteurs extérieurs (voir tableau 3).

La question de la «saisie» des créatifs - c'està-dire la manière dont se fait leur implication sur un nouveau projet - donne lieu à des modèles différents. Le premier, l' «auto-saisie», se rencontre dans l'édition - notamment dans la littérature générale - ou dans la création musicale et, de façon générale, dans les activités artistiques. Dans l'audiovisuel, à côté du modèle traditionnel où le scénariste est à l'origine d'un projet, il y a le modèle où ce dernier peut être embauché pour travailler sur un sujet ou sur un thème particulier ${ }^{8}$. Dans un troisième modèle enfin, le cahier des charges n'est pas fourni par l'entreprise qui développera le projet mais par un client extérieur: on trouve ce modèle par exemple dans le domaine des parfums.

À côté de ces modèles dominants, en émergent d'autres dans chacun des secteurs. D'autre part, des différences apparaissent dans les modèles dominants selon la zone géographique d'implantation ou selon l'époque : c'est le cas par exemple du cinéma, qui fonctionne différemment selon qu'il s'agit du système français ou du système hollywoodien, et différemment par ailleurs dans le système hollywoodien selon qu'il s'agit du système actuel ou de celui des années 1970. Ceci montre que les formes d'organisation de la création relèvent aussi d'un facteur conventionnel.

Derrière le modèle général des processus de création cohabitent des formes d'organisation industrielle variées. Trois modèles types idéaux mais non exhaustifs se dégagent: le modèle «romantique», le modèle intégré et le modèle externalisé (voir tableau 4). Dans le premier d'entre eux - romantique -, dont l'édition littéraire est l'archétype, la création est presque intégralement externalisée. Dans le deuxième - intégré -, elle est complètement assumée à l'intérieur de l'entreprise qui prend en charge la commercialisation. Quant au dernier, si la création est externalisée, c'est dans le cadre de commandes passées à une entreprise spécialisée en la matière.

En termes de management, les problématiques varient selon le modèle - choisi ou imposé. Dans les organisations où les projets, au niveau de la sélection et de la formalisation finale, sont développés spontanément, en dehors de toute structure,

Tableau 3 - Les modèles de «saisie » des créatifs

\begin{tabular}{|lcccc|}
\hline & Projet d'auteur & Projet de producteur & Commande interne & Commande externe \\
Entité instigatrice du projet & Créateur & Entreprise de création & Client interne & Client externe \\
Exemples & Édition littéraire & Jeu vidéo & Automobile & Parfums \\
\hline
\end{tabular}

Tableau 4-Trois modèles types idéaux d'organisation industrielle de la création

\begin{tabular}{|lcccc|}
\hline & & \multicolumn{2}{c|}{ Étapes du processus } \\
& Initiation & Développement & Validation & Exemples \\
et cadrage & et formalisation & et labellisation & \\
Modèle « romantique " & Créateur & Créateur & Créateur & édition littéraire \\
Modèle intégré & Créateur & Équipes de création en interne & Créateur & haute-couture, design automobile \\
Modèle externalisé & Marque & Équipes de création externes & Marque & parfumerie, publicité \\
& & & & \\
\end{tabular}

8. Thomas PARIS, Le droit d'auteur. L'idéologie et le système, Paris, PuF, 2002. 
l'entreprise doit s'organiser pour détecter ces projets et les sélectionner de manière à s'assurer un flux régulier de créations : les structures d'édition notamment sont dans cette situation. Dans les organisations qui intègrent les créatifs, il s'agit de détecter ceux-ci, de les former, les fidéliser, d'organiser le renouvellement de leur inspiration et de gérer leurs échecs. Dans les organisations qui sous-traitent la création, il s'agit de gérer une image de marque. Les choix effectués en termes d'organisation ont toujours une influence sur la créativité et sur la nature de la production finale.

\section{Créateurs, créatifs : quelles particularités?}

Les individus engagés dans des activités de création - créatifs, créateurs, artistes et autres auteurs partagent trois particularités qui traversent les activités de création : le goût pour le renouvellement, l'existence de règles intimes, la fragilité.

La propension au renouvellement. Pour exister, être visible et se distinguer, le créateur doit apporter quelque chose de nouveau, de différent et doit donc à ce titre remettre en cause les cadres établis, se remettre en cause lui-même et entretenir la propension au renouvellement. Il est donc essentiel qu'il ait une connaissance approfondie de ce qui s'est fait et se fait dans son champ : le créateur de mode par exemple, lorsqu'il arrive dans une nouvelle maison, va souvent se plonger dans les archives, animé d'un double mouvement d'inspiration et de dépassement.

Des règles intimes. À côté des règles de toute nature (conventionnelles, culturelles...) qui régissent la plupart des champs de la création, les créateurs ont aussi parfois leurs règles intimes, formalisables ou non, qui définissent, de manière explicite ou non, leur style. Des règles de composition, comme dans les parfums par exemple : il est admis aujourd'hui qu'un parfum est le mariage de notes de tête, de cœur et de queue. Des règles d'harmonie : Alain Senderens s'est construit au fil du temps des règles très précises sur le mariage des vins et des plats, «sa palette de peintre ».

Ego, donc fragilité. Troisième trait caractéristique pour comprendre la nature des activités de création et la manière dont ces activités pourront s'inscrire dans des espaces organisés : l'ego et la fragilité des créateurs. Pascal Nègre ${ }^{9}$ explique à propos des artistes musiciens que la nature même de leur métier, leur imposant une mise à nu et une confrontation permanente au public, accroît leur propension à posséder un ego déjà important et se traduit par une grande fragilité face à l'échec. Patrick le Quément ${ }^{10}$ montre combien il est important d'expliquer aux designers, lorsque leurs projets n'ont pas été retenus, qu'ils ont néanmoins contribué au travail collectif et donc aux projets retenus. Cette gestion des talents se retrouve également dans la sélection de l'équipe de France de football.

\section{Création et structures}

Saisir le lien entre création et structures suppose de comprendre comment ces dernières, qu'elles soient organisationnelles, institutionnelles ou autres, influent sur les processus mis en œuvre dans les activités de création et ce qui en résulte en termes de nature de production. Pour confronter les processus de création aux différents niveaux de structures organisées dans lesquelles ils s'inscrivent, il faut identifier les différentes contraintes propres aux univers organisés - économiques, industrielles, de formalisation... - et les dispositifs par lesquels le bon déroulement de ces processus est garanti malgré ces contraintes.

Une fois identifiés, il s'agit de comprendre comment les projets sont gérés et organisés les temps de divergence et de convergence. Au-delà des projets, les entreprises des industries créatives doivent gérer la création, c'est-à-dire prendre en compte des préoccupations spécifiques aux activités de création: générer des projets nombreux, détecter les talents, gérer leur formation ainsi que leurs échecs, garantir la cohérence d'une ligne éditoriale ou d'une image de marque... Enfin, puisque différents niveaux interviennent dans la constitution des environnements au sein desquels se développe la création, l'angle d'observation s'ouvre jusqu'à introduire le point de vue des politiques publiques: se pose alors la question des dispositifs qui peuvent favoriser la créativité. 
Gérer les projets de création, c'est gérer l'équilibre

divergence-convergence

Un des enjeux dans le management de la création consiste à faire en sorte que les personnes impliquées dans les processus soient très libres dans leur recherche, tout en réintroduisant en permanence des dispositifs pour favoriser la convergence en vue d'aboutir à un projet. Convergence et divergence ne sont pas des étapes successives d'un processus, mais interviennent l'une et l'autre en permanence.

\section{Cadrage}

Le cadrage introduit d'emblée de la convergence : il délimite le champ dans lequel les créateurs vont effectuer leurs recherches et revêt des formes variées : cahier des charges dans le design, brief dans les parfums, orientations thématiques dans la recherche d'idées de nouveaux services, principes directeurs dans la construction d'un nouveau restaurant, mots-clés ou posters dans la mode, description d'une ambiance dans le jeu vidéo, histoire dans la haute couture ou le jeu vidéo... Ces dispositifs de cadrage, loin de brider la créativité, peuvent au contraire la favoriser : une directrice de collections de vêtements de mode a ainsi vu la créativité de ses équipes augmenter lorsqu'elle a introduit un cadrage précis pour le dessin des collections. La difficulté du cadrage tient donc à son ambivalence puisqu'il limite l'ouverture et favorise en même temps la créativité : pour le fondateur d'une agence de publicité, un cadre précis n'est ainsi jamais une entrave à la créativité mais ce sont les contraintes imposées par une exigence de formatage qui la limitent.

Le cadrage constitue certes une étape très importante mais n'est pas défini une fois pour toutes. Il est précisé à chaque étape de formalisation à partir des réalisations intermédiaires. Car la formalisation est l'autre grande difficulté du cadrage : celui qui lance le projet exprime son attente en décrivant des sensations, des ambiances, des goûts... avec un arsenal d'outils très rudimentaire - des mots et des images. Deux cas de figure sont possibles: le cadrage peut être volontairement ouvert de manière à laisser libre cours à la créativité, ou bien correspondre à la vision que le créateur a du produit auquel il souhaite arriver.

Le premier cas - cadrage ouvert - se rencontre souvent lorsqu'il est défini par des gestionnaires et non des créatifs : en radio, par exemple, le respon- sable des documentaires d'une grande station nationale propose un cadrage en termes de thématique ; mais c'est aussi le cas lorsqu'il est défini par un créatif qui développe une collection et non un produit: dans une entreprise de haute couture, le cadrage est proposé par le créateur de façon très ouverte parce qu'il a en tête non pas la collection dans ses détails, mais son esprit général.

Dans le second cas de figure, le cadrage correspond à la vision du produit auquel le créateur souhaite arriver : l'objectif est alors de mettre en œuvre cette vision même si la formalisation implique nécessairement de la divergence (inspiration) de la part des collaborateurs artistiques : c'est le cas par exemple dans la réalisation de films.

\section{Divergence}

La gestion de la divergence mobilise de nombreux dispositifs ou formes d'organisation, qui exploitent le rôle joué par l'ouverture à des cultures différentes dans les processus créatifs. Immersion dans des univers très variés, confrontations avec d'autres univers (voir encadré) ont pour but de permettre aux créatifs de sortir de leurs cadres de pensée et de remettre en cause les contraintes qui peuvent s'imposer à eux.

Quand la création ne constitue qu'une partie de l'activité de l'entreprise (Renault, Studio Créatif de France Télécom...), les créatifs sont souvent isolés du reste de l'entreprise pour être le plus possible à l'abri de toutes les contraintes - techniques, marketing... - dans la phase de divergence. C'est aussi une façon de les protéger des différentes formes de

\section{Quelques exemples de dispositifs}

Dispositif d'immersion - Les créatifs en agence de publicité parcourent les revues consacrées aux thématiques « mode, maison, arts... " pour se plonger dans ces univers très variés; des missions sont organisées lors d'événements culturels dans le cadre de «missions tendances » (Renault), dans des expositions ou musées (Kalisto, Senderens, Renault); les designers de Renault ont la possibilité de partir se ressourcer dans des centres satellites au centre de grandes villes culturelles.

Dispositif de confrontation - Dans le restaurant qu'ils ont réalisé ensemble, Alain Ducasse et Chanel ont effectué un travail de "passerelles » appuyé sur des plongées réciproques dans leurs univers respectifs ; chez Renault, les designers ont travaillé avec des concepteurs de montres ou de bateaux pour être confrontés à d'autres façons de penser et, peut-être, ouvrir leur démarche créative; dans la formation des designers de Strate Collège ou au Studio Créatif de France Télécom, la rencontre et la confrontation interdisciplinaires sont perçues comme des sources de créativité. Le fondateur de l'agence de communication La Chose mobilise, dans les réunions de créativité, des jeux de cartes qui, en imposant des contraintes originales, aident les participants à sortir de leurs cadres de pensée. 
pression car, selon Brigitte Romagné, pression et création ne font pas bon ménage. Le responsable du design de Renault revendique et défend le droit à ses designers d'être des bad boys situés dans la transgression. L'exclusion de toutes les contraintes est aussi favorisée chez l'agence de publicité BETC où la responsable demande à ses équipes de ne valoriser ni le relationnel avec le client, ni la rapidité, mais un travail dont ils puissent être fiers. D'autres dispositifs, construits autour de l'idée de confrontation, contribuent à organiser la divergence : le travail en équipe qui permet, selon une ancienne directrice de création dans le parfum, d'éviter la myopie du créateur; l'organisation de manière plus ou moins formelle de temps pour échanger : brainstorming dans la publicité, réunions jalonnant l'avancée des projets dans la création de restaurants ou la réalisation de films d'animation.

Une division du travail trop stricte ne semble pas propice à la divergence car, les processus de création n'étant pas linéaires, les phases d'exécution et de mise en forme, ainsi que la confrontation permanente entre les concepts et les formes, peuvent être source d'idées. Ces dispositifs, qui ont des vertus à la fois pour la divergence et pour la convergence, montrent en filigrane que la créativité est infinie. L'activité de création s'oppose aux processus de décision dont les théories ont montré qu'elles reposaient sur la recherche d'un optimum ou d'une solution satisfaisante ${ }^{11}$. Reconnaître cette spécificité des activités de la création, c'est reconnaître l'importance du facteur temps dans les processus créatifs et, au-delà, que l'évaluation des activités de création ne peut se faire selon les modalités d'évaluation des autres activités.

\section{Convergence}

La convergence intervient dès que le processus de création est lancé : réunir une équipe, c'est déjà orienter le projet dans la mesure où le choix de l'équipe, du créateur, oriente la création dans certaines directions.

L'organiser ensuite, c'est faire en sorte que le projet débouche sur une production dans des délais déterminés en prenant en compte différents types de contrainte : faisabilité technique et économique, format, ergonomie. L'intégration de ces contraintes repose sur deux modalités différentes. D'un côté, des équipes intégrées qui fonctionnent en ateliers, dans lesquels se confrontent les différents métiers, donc les contraintes qu'ils représentent. Dans ces ateliers, le processus de convergence-divergence se joue en permanence : c'est le cas par exemple dans le jeu vidéo, où informaticiens et créatifs, impliqués ensemble sur les projets, confrontent sans cesse leurs idées à la question de la faisabilité technique et économique. D'un autre côté, les créatifs sont isolés et les contraintes interviennent à un certain moment du processus. C'est ce qu'on trouve dans le bâtiment par exemple: les architectes dessinent le projet avant de le confier aux entreprises chargées de le réaliser. Or, pour des questions d'efficacité du processus, on constate que les modalités de travail évoluent et que de plus en plus les architectes travaillent davantage avec les entreprises ${ }^{12}$.

Le choix de l'une ou l'autre de ces modalités aura tendance à favoriser l'efficacité ou la créativité, comme le montre l'exemple des groupes Alain Ducasse et Chanel, à travers la création d'un restaurant commun : alors que le premier a tendance à formaliser les contraintes liées au métier de restaurateur, la création de Chanel fonctionne dans le mode du secret et de l'événementiel. Cette opposition de cultures «atelier/secret» s'explique aussi par la nature des métiers en cause, en particulier dans la haute couture, le rythme connu des collections fait que la visibilité des maisons tient à la surprise qu'elles créeront par le contenu des collections mêmes.

Dans les projets de création qui impliquent des collectifs - cas les plus fréquents -, la convergence consiste aussi à faire en sorte que les différents participants aient une vision du produit allant dans le même sens. Expliciter des attentes précises et de communiquer autour de la création est une difficulté. Trois facteurs favorisent la convergence des vues et des apports: les échanges nombreux, les mises en forme intermédiaires, et le temps. La notion d'acculturation est très importante : les mots étant insuffisants par eux-mêmes, ils prennent sens dans un contexte de culture commune qui se crée dans le temps et suppose que les références soient partagées, voire le langage commun. Dans le restaurant d'Alain Senderens, ce partage se fait lorsque le chef envoie ses seconds dans des expositions, leur conseille des livres, pour faire en sorte qu'ils appréhendent son univers créatif dans sa globalité. Une ancienne directrice de création dans les parfums a,

11. J. G. March et H. A. Simon, Organizations, New York, John Wiley \& sons, 1958 (trad. fr. Les Organisations, Paris, Dunod, 1974).

12. T. PARIS, «Le cas de Bouygues Habitat. Le projet de logements "René Villermé" », dans S. BEN MAHMOUD-JouINI (sous la dir. de), Coconception et savoirs d'interaction, Paris, Plan urbanisme construction architecture (PUCA), 2003, p. 211-225. 
elle, développé un langage pour permettre aux personnes en charge de cadrer la création d'être très précises.

À cet égard, les petites structures favorisent peutêtre la compréhension réciproque car s'y développe davantage une culture commune. De même, cela explique peut-être l'importance de la figure du duo manager/créatif dans les activités de création, dont Domenico De Sole/Tom Ford ou Yves Saint-Laurent/Pierre Bergé sont de bons exemples. En s'appuyant sur une relation forte, le duo internalise les difficultés de communication autour de la création. Autre facteur important enfin, la durée des équipes, qui permet la construction d'une culture commune et favorise le travail ensemble et la communication. Lorsque la création repose sur des organisations non permanentes, comme c'est le cas dans le cinéma ou le théâtre, la constitution d'une équipe capable de travailler voire de créer ensemble demande un certain temps. Ainsi, l'ancien responsable de Kalisto évalue ainsi à plus d'un tiers du budget de développement d'un jeu vidéo consacré au travail de construction de l'équipe.

Choisir à chacune des étapes de mise en forme constitue un autre volet de la convergence. Le choix peut intervenir en amont du processus de création, dans sa forme organisée, ou en aval. Dans ce cas, la convergence s'organise autour de dispositifs de filtrage et de sélection des projets reposant sur la confrontation de points de vue des personnes de l'entreprise ou non, ou sur des critères de différents ordres, économiques ou techniques.

Deux temps, des allers-retours permanents de l'un à l'autre : convergence et divergence traduisent la dualité entre deux mondes, celui de l'inspiration et celui de l'organisation. L'un est fait de liberté, l'autre de structures qui sont autant de contraintes. La manière dont leur équilibre est géré, et plus généralement la manière dont les structures des univers organisés sont introduites dans les processus de création, ont un impact sur la production de ces processus.

\section{La gestion}

\section{dans les entreprises de création}

La créativité se gère au-delà des projets de création. La capacité d'une entreprise à organiser l'abondance des projets en amont, à identifier, for- mer, gérer, fidéliser les talents, à se doter d'outils de pilotage économique..., autant de d'éléments structurants qui influeront sur la création produite.

\section{La gestion de l'abondance}

L'abondance est une nécessité inhérente aux industries créatives, qui tient à la propriété nobody knows $^{13}$. D'un côté, pour alimenter les réseaux de diffusion et amortir l'ensemble de leurs coûts, les structures de production et de diffusion ont besoin d'un flux régulier de productions; de l'autre, la création n'est pas une activité au résultat prévisible. L'incertitude quant au résultat se traduit par une nécessaire surabondance de projets par rapport aux besoins du marché et par la mise en place de dispositifs de sélection à toutes les étapes, laquelle sélection peut intervenir en amont, au cours ou à la fin des projets.

L'abondance peut être organisée au sein des entreprises ou au contraire laissée au marché. Dans les secteurs de l'édition, de la musique ou du cinéma, elle intervient en amont des entreprises : ce sont les aspirants talents qui la prennent en charge en créant spontanément et en soumettant leurs créations aux entreprises de production. Dans l'industrie automobile ou des télécommunications, elle est organisée en interne : les designers de Renault par exemple participent à des compétitions internes sur chaque projet.

Il en est de même pour la sélection : ce sont soit les entreprises soit des institutions qui en ont la charge. Elles peuvent porter sur des produits à des stades de développement différents: des projets dans le cinéma (scénarios) ou des produits finis dans l'édition (manuscrits) par exemple. La gestion de l'abondance et de la sélection par les entreprises peut se situer à chaque étape : en amont des projets où elles en déterminent un certain nombre sur lesquels travailler; en cours de projet à travers les choix qu'elles font à chaque étape de mise en forme; en fin de projet lorsqu'il s'agit de créations qui leur arrivent du marché. Selon que l'abondance a été organisée en interne ou prise en charge par le marché, les dispositifs pour organiser le choix diffèrent. Lorsque l'abondance est issue du marché, les entreprises doivent être capables de susciter des projets et des talents et de les sélectionner. Les dispositifs organisationnels mobilisés peuvent avoir une influence sur la nature de la création. Dans

13. Énoncée par Richard Caves. Voir R. E. CAVES, Creative industries. Contracts between Art and Commerce, Cambridge (Mass) et Londres, Harvard University Press, 2000. 
l'édition par exemple, les résultats seront différents selon que l'on recourt à un comité de lecture ou que le choix est confié à l'éditeur. La première solution amènera peut-être une production plus consensuelle, moins audacieuse et pourra aussi se traduire par des jeux de négociation qui déborderont du strict cadre de l'évaluation d'un texte. La seconde, en éliminant les impératifs de justification, mettra plus en avant les coups de cœur, mais dans le même temps, engendrera plus de risque, par exemple que l'éditeur soit pris par un rapport de séduction avec l'auteur.

La nécessaire abondance organisée dans les industries de la création soulève la question de sa gestion. Pour David Kessler ${ }^{14}$, c'est une des problématiques principales rencontrées par le cinéma français aujourd'hui qui, s'il a su organiser l'abondance nécessaire à son dynamisme, n'a pas encore mis en place les processus pour arrêter un certain nombre de projets, et opérer une sélection plus drastique. La difficulté est en effet double: sur le plan financier, il n'est jamais aisé d'arrêter un projet sur lequel des investissements ont été réalisés; sur le plan humain, arrêter un projet est vécu comme un échec pour les personnes qui y ont été impliquées. Autre exemple donné par Pierre Chevalier ${ }^{15}$, Arte et France Culture : dans le premier, la sélection passe par différents comités - comité de lecture, commission des programmes... - alors que dans le second, tout se passe dans la relation entre le responsable d'une case de la grille de programmes et les producteurs. Chaque dispositif, s'il limite le risque, est un niveau de formatage supplémentaire de la création.

La composition des commissions, le profil des personnes en charge des sélections... autant de logiques - esthétique, économique, importance de la prise de risque - qui orientent le type de production. La question du mode de sélection est étroitement liée à la manière dont l'entreprise accompagne ensuite les projets : l'expérience confirme que des choix assumés par une personne sont assez cohérents avec un accompagnement très fort, alors que des choix organisés collectivement s'accordent avec un accompagnement plus faible, une partie du risque ayant été gérée par la dimension collective du choix.

La nécessaire organisation de l'abondance implique des dispositifs de sélections, voire dans certains cas l'accompagnement des postulants dont le projet n'a pas été retenu. Elle suppose également des dispositifs de pilotage économique adaptés à la configuration dans laquelle le « déchet» fait partie intégrante de la création.

\section{La gestion des talents}

La détection et la sélection des projets sont souvent étroitement liées à la détection des talents. Or le recrutement des créatifs ne peut pas reposer sur des critères formels car le talent s'évalue subjectivement, ce qui contribue à constituer une catégorie particulière de talents chargés de les détecter et les sélectionner.

De nombreux canaux sont mobilisés pour identifier des talents potentiels, la règle étant en la matière de faire feu de tout bois : concours et festivals (jeunes talents à Cannes, festivals de courtsmétrages, prix divers...), candidatures spontanées ou soumissions de projets, réseaux formels ou informels utilisés pour les détecter à l'occasion d'un contact, d'un casting... Trois types de dispositifs existent : les têtes chercheuses, individus missionnés par une entreprise pour sillonner les lieux d'expression des talents et les recruter - c'est la façon de détecter dans le sport professionnel; les agents indépendants, qui assurent le rôle d'interface entre les talents et les entreprises et prennent en charge la détection; les réseaux informels, dispositif qui fonctionne dans tous les secteurs.

À côté de ces trois modes de détection actifs, on trouve des modes «passifs » auxquels recourent les entreprises dotées d'une image forte qui laissent arriver projets et talents, sachant qu'une première sélection est effectuée par les postulants eux-mêmes qui s'autocensurent en raison de cette image forte. Ce mode de détection implique que l'entreprise ait la capacité d'étudier chacun des projets arrivés. La capacité à rester ouvert sur l'extérieur, difficile à gérer du fait de l'abondance des projets qui arrivent, est une clé pour les entreprises qui concilient exigence éditoriale et image forte.

Gérer des créatifs, c'est prendre en compte la dimension «échec», composante intégrante des métiers de création, à la fois parce que le succès d'une production n'est jamais garanti et parce que les filières de création fonctionnent sur un modèle de sélectivité très importante. Lorsque les créatifs font partie d'une entreprise, celle-ci doit gérer ce sentiment d'échec. Les petites structures, où peut

14. Lorsqu'il était directeur du CNC.

15. Ancien directeur de l'unité fictions d'Arte France. 
s'instaurer une grande proximité entre talents et gestionnaires, sont, là encore, une façon d'y répondre. Pascal Nègre compare ainsi un label, structure élémentaire au sein des maisons de disques, à un village. Au design de Renault, la gestion de l'échec (ou du ressourcement) se fait en envoyant les designers dans des centres-satellites, et aussi grâce aux «concept-cars », véhicules non destinés à la production, qui permettent aux designers de s'exprimer sur des projets plus nombreux.

\section{L'évaluation économique de la création}

Cette nécessaire abondance de l'offre explique l'important besoin en financement des activités de création, qui est leur difficulté intrinsèque. Ainsi, pour aller au bout de la logique de production d'idées en masse, France Télécom devrait consacrer des ressources infinies à son Studio Créatif! Dans le jeu vidéo, la question du financement est rendue plus critique par le fait que les studios doivent absorber tous les cinq ans les coûts liés aux sauts technologiques. Cette question des ressources est d'autant plus importante qu'elle se heurte à celle de l'évaluation des organisations créatives, qui ne concrétisent qu'une partie des projets explorés.

Si la phase de convergence, qui débouche sur des productions appelées à rencontrer le marché, s'accorde avec des critères d'évaluation économiques, elle ne peut être déconnectée de la phase de divergence, de production d'idées, laquelle est plus difficilement évaluable. Que ce soit au sein d'une entreprise ou d'un groupe industriel, comme le montrent les exemples de France Télécom, d'Actes Sud ou d'Universal Music, ou au niveau d'un secteur comme celui du cinéma, une évaluation économique classique, axée sur les notions de produit et de chiffre d'affaires, paraît inadaptée à la réalité des entreprises de création, qui s'accorde mieux avec les notions de « collectif », de catalogues et de résultats de long terme.

La capacité des entreprises de création à faire passer au second plan - second par rapport à des choix éditoriaux - les impératifs financiers à court terme apparaît même comme fondamentale pour plusieurs entreprises de création. Le cas de Nova est en cela emblématique : la contrainte imposée par le propriétaire de la radio à ses équipes n'est pas de gagner de l'argent mais de ne pas en perdre, l'essentiel étant que l'entreprise ne perde pas son âme. Cela peut se traduire par l'importance accordée à l'indépendance, mais une indépendance qui doit composer avec l'importance des besoins de financement, liée à la nature des activités de création.

Apparaît ici une difficulté. D'un côté des petites structures, pas en mesure de générer et de supporter l'abondance nécessaire dans la création. De l'autre, de grandes entreprises, c'est-à-dire des règles, des routines, des barrières, des contraintes, soit autant d'entraves à une liberté créatrice. Certes, la création peut être isolée du reste de l'entreprise, regroupée dans de petites structures au sein de grandes entreprises, mais tout n'est pas si étanche, et il reste que la créativité sera toujours plus à l'aise dans des structures petites et fragiles.

Derrière ce constat, se pose la question plus générale du rapport qu'entretiennent les structures avec la créativité, et derrière cette question, une autre, plus opérationnelle : "Que signifie construire un environnement propice à la créativité ?»

\section{Quels espaces pour la création?}

L'organisation des structures existantes - à savoir les contraintes financières, marketing, techniques qui s'imposent aux processus de création formate donc la création. Mais en outre, parce qu'elles n'évoluent pas sur un terrain vierge, parce qu'elles ont des clients, au sens le plus large du terme, les organisations peuvent avoir tendance aussi à reproduire le passé et à limiter, en partie, le renouvellement créatif.

L'habitude rassurant, la référence aux succès passés minimisant les risques, ces structures peuvent parfois devenir des structures mentales, qui s'imposent comme règles avec le temps : «les gens du marketing ont tendance à voir avec un rétroviseur », dit le responsable d'une maison de haute couture. De même, la nouveauté est difficilement comprise par les structures existantes : présenter le pitch $^{16}$ de La marche de l'empereur pour réunir le budget s'est vite avéré voué à l'échec, parce que le format ne permettait pas de rendre ce qui ferait le film. «C'est l'histoire des manchots sur la banquise... » La campagne de communication pour Air France, chez BETC, a connu la même difficulté, dès lors que l'agence avait décidé de travailler sur la lenteur, c'est-à-dire sur quelque chose de radicalement nouveau dans le monde de la publicité.

Pour identifier les espaces de créativité, trois types de situations ouvrant le champ de la création

16. Le pitch d'un film doit en quelques mots susciter l'intérêt pour le film : il est utilisé comme outil de promotion. 
ont été identifiés : la remise en cause des contraintes financières par des entreprises, l'émergence de nouveaux espaces de diffusion, l'apparition d'une nouvelle technologie.

\section{Remise en cause des contraintes financières}

Dans un souci de se différencier, ou pressées par la contrainte financière elle-même, ou bien encore parce qu'elles sont engagées dans une forme de mécénat, certaines entreprises de création sont amenées à remettre en cause les contraintes économiques traditionnelles. Cette remise en cause peut s'avérer féconde : Alain Senderens, en décidant de casser les codes de la grande cuisine et de sortir du modèle dominant des restaurants trois étoiles, remet en cause les contraintes financières antérieures et s'impose de nouvelles contraintes de coût pour réduire significativement le prix de ses prestations. Ceci s'est traduit par une sortie du star system puisque, au lieu de s'appuyer sur les produits ou vins de luxe, il est obligé d'être beaucoup plus actif dans la recherche de produits, notamment de vins méconnus et de qualité - de nouveaux «talents »et donc de se montrer plus créatif pour les mettre en valeur. Par ailleurs, la petite taille ou la «pauvreté » obligent à trouver de nouvelles façons de travailler, de promouvoir leurs créations : c'est le cas des petits labels.

Autre type d'ouverture, le modèle de Nova montre comment l'objectif financier de ne pas perdre d'argent, imposé par le propriétaire aux équipes, peut aussi libérer la créativité. Une certaine prise de risque devient possible dans les choix en matière d'animateurs et de musiques libérés des contraintes d'audience maximale immédiate pesant sur l'ensemble des radios, ce qui permet d'avoir un positionnement d'avant-garde et de découvreur.

Quant au VIA, organisme financé par une taxe affectée qui est prélevée auprès de l'ensemble des industriels de l'ameublement, son budget n'est pas directement connecté à son activité, ce qui lui permet de jouer un rôle d'aiguillon pour la créativité du design dans l'ameublement et le mode de vie en impulsant des travaux autour de thèmes ( la chaise »), en finançant des jeunes designers, en exposant leur travail.

\section{De nouveaux espaces de diffusion}

L'affirmation de nouveaux espaces de diffusion constitue un appel d'air pour la création. Ainsi, lorsque Pierre Chevalier mit en place sur Arte une collection orientée potentiellement vers le cinéma et la télévision, il ne disposait que d'un budget limité et fut donc conduit à recruter de jeunes réalisateurs avec peu d'expérience, ce qui fit venir au cinéma une nouvelle génération de réalisateurs. Un festival comme celui de la bande dessinée d'Angoulême, indépendant des professionnels et donc obligé de se renouveler et de se situer à l'avantgarde, est extrêmement dynamique vis-à-vis des jeunes talents en leur offrant un lieu d'exposition et en suscitant des vocations SEQARABIC.

Dans la structuration des filières de création, tous les acteurs qui offrent un nouvel espace d'expression et de visibilité peuvent constituer un facteur de créativité : le succès de la bande dessinée francobelge est lié à l'émergence de nouveaux supports de diffusion, à savoir les revues qui ont offert une tribune aux jeunes talents ${ }^{17}$. Chaque institution, par son fonctionnement, son mode de sélection, les réseaux dans lesquels elle s'imbrique, constitue un filtre particulier pour les talents et les créations. Les institutions de formation et de labellisation ont ainsi un poids considérable dans la structuration de la création. L'affirmation de nouvelles institutions, lorsqu'elles reposent sur d'autres modes de sélection, constitue alors une ouverture comme c'est le cas de Cube pour la création numérique ou le festival de Pâques à Deauville en musique.

Enfin, l'établissement de passerelles entre modes de diffusion proches, comme la télévision et le cinéma, peut être également une source de créativité. Les passerelles amènent en effet les créateurs à se confronter à d'autres contraintes, chaque mode de diffusion ayant tendance à imposer un ou des formats dans la création. C'est aussi un moyen d'organiser mieux la formation, parce qu'elles permettent aux talents de passer de formats moins coûteux à des formats plus coûteux. L'apparition d'un nouveau format, même s'il peut attirer des talents venus des formats proches, peut aussi être l'occasion d'une ouverture, comme le montrent les exemples des formats courts à la télévision ou sur internet.

\section{L'émergence de nouvelles technologies}

De façon générale, les technologies novatrices permettent l'émergence de nouveaux espaces et créent de multiples opportunités pour favoriser la créativité. Elles obligent à remettre en cause les compétences des créateurs, de ceux qui sont instal-

17. Jean-Marc Thévenet, ancien directeur du Festival de la bande dessinée d'Angoulême. 
lés, tous se trouvant à égalité vis-à-vis d'elles. Elles peuvent d'autre part faire baisser les coûts ou les déplacer, comme on le constate avec le numérique, et donc permettre l'abaissement des barrières à l'entrée à la création. Cet appel d'air permet à de nouveaux acteurs de se faire une place, de s'approprier les moyens de création, de proposer de nouvelles formes de création. Les marchés existants s'en trouvent bousculés, et c'est l'ensemble des structures, et des règles pour la création dont elles sont porteuses, qui est remis en cause.

\section{CONCLUSION}

Création et organisation reposent sur des modes de fonctionnement a priori opposés mais conci- liables dans la perspective d'un pilotage de la créativité. La création peut être «gérée » puisque derrière les processus de création des mécanismes interagissent avec les organisations. Dans des univers organisés, la création peut être gérée sous réserve d'une analyse de ces univers, des dispositifs qui les composent, et des effets qu'ils ont ou auront sur la création. Elle se gère aussi de façon globale dans la mesure où ces univers s'inscrivent les uns dans les autres: les projets dans les entreprises, les entreprises dans des industries. Si certaines configurations d'organisation de la créativité font modèles, elles ne peuvent pas à ce stade être considérées comme des one-best way. Car, du fait de leur tendance à formater la création, plus les structures sont variées, plus grande sera la liberté créative.

\section{État de la recherche}

Le sociologue Howard Becker a publié en 1974 "Art as collective action" ", article qui démontait la vision romantique de la création et intégrait les activités artistiques dans le champ des actions collectives. Ouvrant la voie des travaux relevant des « approches institutionnelles de l'art ", ces recherches développaient le fait qu'un bien culturel était aussi le produit d'une organisation socio-économique sur lequel pesaient des contraintes variées: financières et techniques ${ }^{2}$, luttes d'institutions pour accéder à la reconnaissance ${ }^{3}$, conventions propres à chaque monde de l'art ${ }^{4}$, pratiques contractuelles ${ }^{5}$, environnements législatifs ${ }^{6}$, structures industrielles ${ }^{7}$.

\section{Travaux au niveau individuel et organisationnel}

Des recherches ${ }^{8}$ se sont multipliées récemment sur la "créativité organisationnelle ", afin d'identifier les leviers à disposition des entreprises pour améliorer leur créativité. Dans une perspective psychosociologique, elles prennent le groupe comme objet pour étudier les facteurs de sa créativité et la manière dont le collectif interfère avec la capacité des individus à produire des idées.

Dans une tout autre approche ${ }^{9}$, les travaux sur les outils de gestion mettent en évidence l'existence d'une technologie invisible qui
" engendre souvent mécaniquement des choix et des comportements échappant aux prises des volontés des hommes ", pouvant conduire "les organisations dans des directions voulues par personne et les rendent même rebelles aux efforts de réforme ». Une approche plus large ${ }^{10}$ analyse les décisions des acteurs individuels au sein d'espaces organisés pour constituer les théories de l'organisation focalisées sur les notions de pouvoir ${ }^{11}$, de règles ${ }^{12}$, de rationalité limitée dans les processus de décision ${ }^{13}$.

Des recherches plus récentes sur les processus et le management d'innovation établissent des spécificités cognitives de l'activité de création et d'innovation par rapport à la décision ${ }^{14}$.

\section{Travaux au niveau territorial et sectoriel}

D'autres recherches abordent des questions relatives à l'impact de " macro-structures » sur la créativité d'une industrie, d'un territoire ou d'une nation. Si la question de la mise en place d'un environnement favorable à la création n'est pas posée de manière globale dans les recherches académiques, elle fait écho à une question semblable qui fait l'objet de travaux depuis un certain nombre d'années. La problématique des «systèmes nationaux d'innovation ",

1. H. S. BECKER, "Art as collective action", art. cité.

2. Michael BaXAndall, Painting and Experience in Fifteenth Century Italy, Oxford, Oxford University Press, 1972

3. Pierre Bourdieu, Les règles de l'art, Paris, Le Seuil, 1992.

4. H. S. BECKER, "Art as collective action", art. cité et Art Worlds, op. cit.

5. R. E. CAves, Creative industries..., op. cit.

6. T. PARIS, Le droit d'auteur. L'idéologie et le système, op. cit.

7. Pierre-Jean BENGHOZI et Thomas PARIS, "Analyzing distribution to understand the markets of cultural goods", 8th International Conference on Arts \& Cultural Management, HEC Montréal, 3-6 juillet 2005.

8. Theresa M. AmABILE et al., "Assessing the Work Environment for Creativity", Academy of Management Journal, 1996, vol. 39, n 5, p. 1154-1184, et "How to kill creativity", Harvard Business Review, septembre-octobre 1998. Cameron M. Ford et Dennis E. GiolA, Creative Action in Organizations. Ivory Tower Visions and Real World Voices, Sage Publications, 1995.

9. Michel BerRY, Une technologie invisible, Paris, École polytechnique, Centre de recherche en gestion, 1983.

10. Gilles CABRIDAIN, Apports et limites de l'instrumentation financière dans l'émergence de la gestion par projet dans l'industrie automobile, Palaiseau, École polytechnique, 1988 (thèse de doctorat).

11. Michel Crozier, Le phénomène bureaucratique, Paris, Le Seuil, Paris, 1963. Michel Crozier et Ehrard Friedberg, L'acteur et le système, Paris, Le Seuil, collection « Points », 1977.

12. Jean-Daniel ReYNAUD, Les règles du jeu. L'action collective et la régulation sociale, Paris, Armand Colin, Paris, 1989 (3édition 1997$).$

13. Herbert A. Simon, Administrative Behavior, New York, The Free Press, 1945 et id., "A behavioral model of rational choice", Quarterly Journal of Economics, février 1955, p. 99-118. James G. MARCH et Herbert A. SimON, Organizations, op. cit.

14. Armand Hatchuel, Pascal Le MASson et Benoît WEIL, Les processus d'innovation. Conception innovante et croissance des entreprises, Paris, Hermès Science Publication, 2006. 
Graphique 1-Une problématique transverse mobilisant des courants de recherche variés

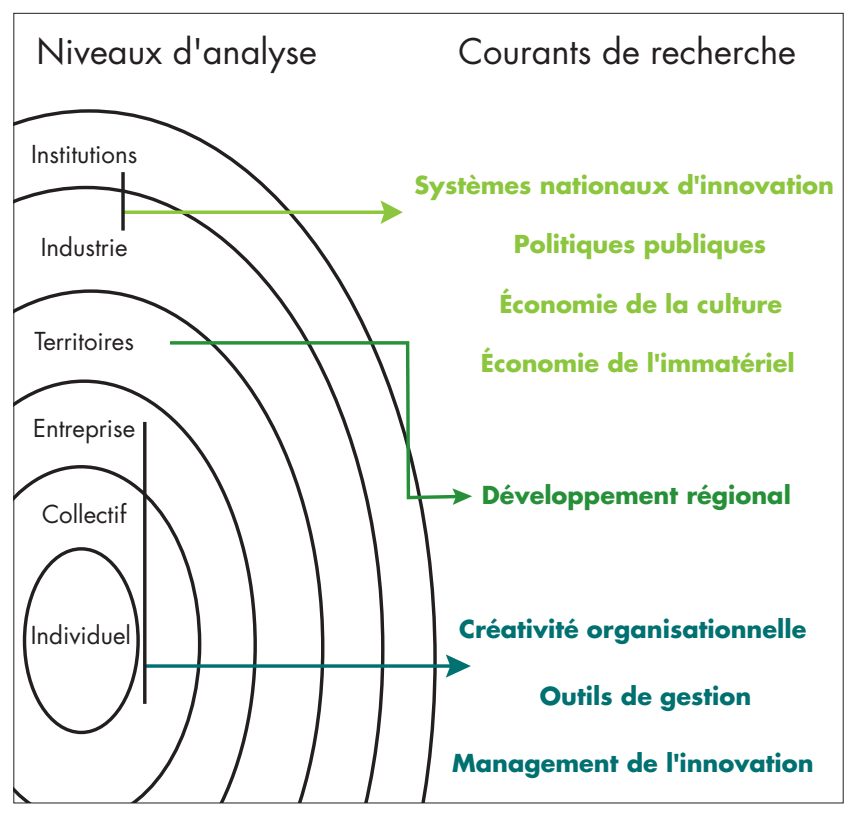

qui est le pendant pour l'innovation du volet «action publique » de notre questionnement, étudie les environnements institutionnels dans la manière dont ils favorisent ou non l'innovation à l'échelle nationale ${ }^{15}$.

Certains théoriciens de l'économie du développement régional ont observé l'influence de facteurs propres aux caractéristiques de l'environnement géographique - démographiques, sociologiques, culturels - pouvant influencer le dynamisme créatif d'un territoire, c'està-dire sa capacité à attirer des « créatifs » (creative class) et à générer des projets nouveaux ${ }^{16}$.

Dans cette perspective, il faut souligner l'initiative prise au RoyaumeUni par les pouvoirs publics de se doter d'une forme d'observatoire des industries créatives dans le cadre d'une politique les affichant comme une priorité ${ }^{17}$.

\section{Une approche transversale}

Le choix d'embrasser ces différents niveaux d'analyse pour adopter une approche transversale repose sur le constat que l'environnement constitutif de la création est composé de cercles concentriques. II permet en outre de rendre plus fertile la dimension comparative. En effet, les dispositifs organisationnels adoptés dans les différents secteurs pour encadrer la création peuvent s'avérer être soit des conventions sectorielles soit des contraintes propres aux conditions spécifiques des différents secteurs. La transversalité permet ainsi de prendre systématiquement en compte, dans l'analyse des dispositifs, l'ensemble des contraintes propres aux secteurs étudiés, et de démêler, dans les dispositifs organisationnels mis en place, ce qui relève de contraintes, de conventions et de choix.

En outre, cela permet de tenir compte des particularités des industries de la création, qui s'organisent autour d'un continuum entre les organisations et le marché. Primo, elles reposent sur des talents, individus dotés d'une aptitude particulière à la création. La non-substituabilité des personnes ( $A$ List / B List property ${ }^{18}$ ) et l'incertitude sur leur talent imposent une gestion collective du risque de sélection, la collectivité assumant le risque de formation et de présélection. En ce sens, les entreprises sont dépendantes du système de formation mis en place, et les niveaux organisationnel et sectoriel sont imbriqués dans la construction d'un environnement créatif. Secundo, les industries créatives mettent en jeu des structures d'organisation ad hoc, ce qui donne un poids important aux institutions (systèmes réglementaires, organisation de la standardisation des compétences) qui facilitent la circulation des personnes et la constitution de projets pertinents. Tertio, la production de ces industries est immatérielle. Cela se traduit par une importance des effets externes, qui jouent autant dans les phénomènes de valorisation (la valeur d'une œuvre, d'un talent dépendra de la valeur d'autres œuvres ou talents) que dans les problématiques d'appropriation et de propriété intellectuelle.

15. Philippe LAREDO et Frédérique SACHWALD, Le système français d'innovation dans l'économie mondiale: enjeux et priorités, Paris, IfRI, Institut de l'entreprise, 2005.

16. Richard Florida, Cities and the creative class, Routledge, 2004. Id., The Flight of the Creative Class. The New Global Competition for Talent, Harper Business, 2005. Martin KENNEY et Richard FloRIDA, Locating Global Advantage. Industry Dynamics in the International Economy, Stanford University Press, 2003. 17. Ministerial Creative Industries Strategy Group, Creative Industries Mapping Document, Londres, Department for Culture, Media and Sport, 2001.

18. R. E. CAVES, Creative industries..., op. cit.

\section{RÉSUMÉ}

Les activités de création mettent en avant le talent individuel mais reposent aussi sur des dispositifs et des organisations. L'investigation soutenue par les ministères de la Culture et de l'Industrie a pris la forme d'un séminaire Création à l'École de Paris du management. Les résultats portent d'abord sur les processus de création et la manière dont les activités de création peuvent s'inscrire dans des projets et des entreprises dans un double mouvement de divergence et de convergence. On examine ensuite les liens entre création et structures en examinant comment est géré cet équilibre divergence-convergence et sont assumées des tâches spécifiques comme la gestion de la nécessaire abondance créative et, partant, l'échec de nombre de projets, la détection et la sélection des talents. La création s'en trouve à la fois encadrée en même temps qu'elle peut trouver de nouveaux espaces de développement.

\section{ABSTRACT}

Title: Artistic creation organisation, processes and structures.

While creative activities necessarily involve individual talent, they also need the support of systems and organisations. The investigation backed by the Ministries of Culture and Industry took the form of a seminar entitled 'Artistic Creation' held at the Paris School of Management. Its conclusions concerned, on the one hand, creative processes and the way these - in a dual movement of divergence and convergence - tie into projects and enterprises and, on the other, the connections between creation and structures. How can the divergence/convergence equation be resolved? How can specific problems such as management of the requisite creative abundance (and the corollary failure of numerous projects), talent discovery and talent selection be handled? Artistic creation is thus kept within limits while at the same time finding new room in which to develop. 


\section{Questions de culture}

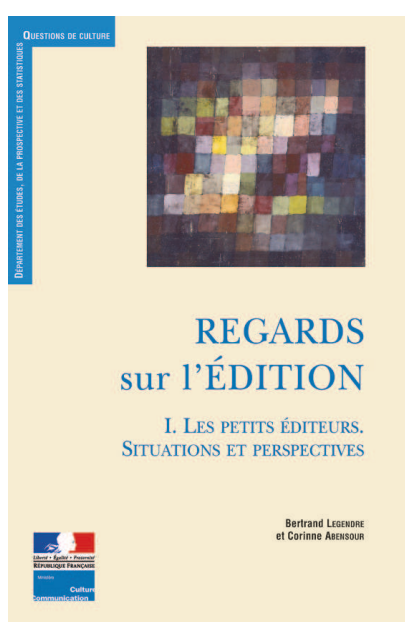

\section{Chiffres clés}
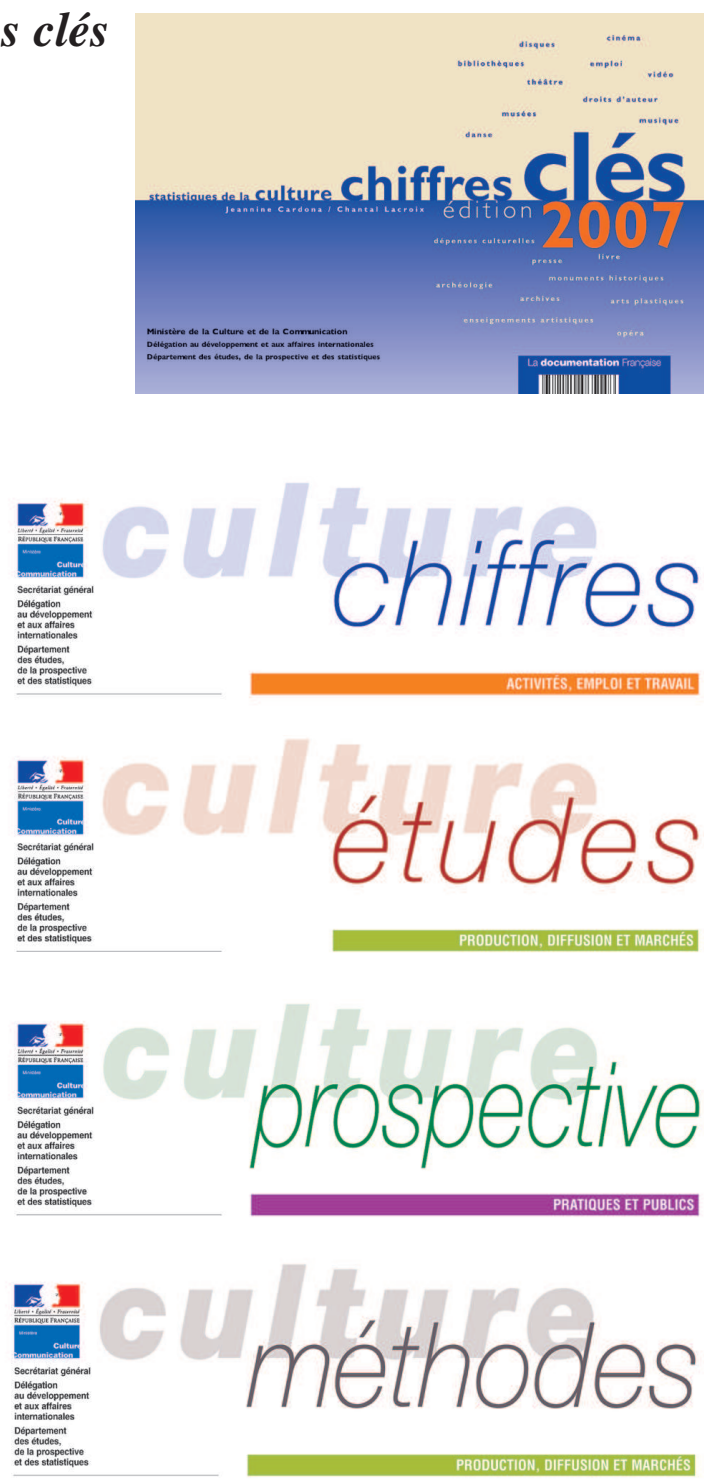

La collection «Questions de culture » (diffusée par la Documentation française) présente une analyse des résultats de travaux d'études ou de recherches en sciences économiques et sociales exécutés dans le cadre du programme du DEPS. Ils visent à rendre disponibles auprès du grand public des résultats ou des approches fermement établis sur les phénomènes culturels mais aussi des recherches innovantes où hypothèses nouvelles et rigueur scientifique s'allient pour penser ces évolutions.

L'annuaire statistique Chiffres clés* présente sous forme de tableaux et graphiques les statistiques relatives à la vie culturelle en France. L'étude aborde le spectre très large du champ culturel (patrimoine, spectacle, enseignements artistiques...) et propose une approche transversale sur les grands thèmes de politique culturelle (emploi, financement, pratiques). L'édition 2007 est publiée sous deux formes: imprimée (en vente à la Documentation française) et téléchargeable sur le site*.

Fidèle à sa vocation de service statistique ministériel, le DEPS présente dans cette collection les résultats chiffrés, les informations statistiques issues de ses travaux. Cette nouvelle collection prend la suite des anciennes (Notes de l'observatoire de l'emploi culturel, Notes statistiques)*.

Cette collection présente les synthèses des études et recherches réalisées par le département, publiées ou non dans la collection «Questions de culture ». Elle succède ainsi à Développement culturel* .

Consacrée à l'investigation d'hypothèses, à des mises en relation originales et à des travaux de prospective sur des champs connus comme sur des terrains plus expérimentaux, cette collection est ouverte à des travaux réalisés au DEPS mais également à des travaux extérieurs (recherches, thèses, etc.) afin de susciter le débat et les échanges au sein de la communauté scientifique*.

Cette collection met à la disposition du public des notes de méthodes dans les domaines qui relèvent des compétences du département (économie, sociologie, statistiques, etc.)*.

\section{* Ces collections sont téléchargeables sur www.culture.gouv.fr/deps rubrique " publications »}

Le DEPS n'assurant pas de diffusion physique de ces documents, nous vous proposons de vous informer régulièrement des parutions par message électronique.

Pour ce faire, merci de bien vouloir nous communiquer votre courriel à l'adresse

contactdeps.ddai@culture.gouv.fr

en indiquant comme sujet du message : "diffusion des collections du Deps". 